Acta Crystallographica Section D

Biological

Crystallography

ISSN 0907-4449

\title{
Instrumentation for synchrotron-radiation macromolecular crystallography
}

$\mathrm{X}$-ray diffraction is an extremely important tool for structure determination of biological macromolecules, to the extent that currently around $85 \%$ of Protein Data Bank entries result from X-ray measurements. Many of these structure determinations use synchrotron radiation for data collection. This article aims to give synchrotron users an overview of the functioning of a synchrotron beamline and how the performance of various instruments combines to allow the collection of diffraction data.

\section{Introduction}

${ }^{a}$ Synchrotron SOLEIL, L'Orme des Merisiers, BP 48 St Aubin, 91192 Gif sur Yvette, France, bESRF, 6 Rue Jules Horowitz, 38043 Grenoble, France, 'IRISA, Campus de Beaulieu, 35042 Rennes, France, and INRA, Avenue des Etangs, 11100 Narbonne, France

Correspondence e-mail: andrew.thompson@synchrotron-soleil.fr

Data collection at the synchrotron involves the measurement of reflection indices $(h \mathrm{kl})$, structure factors and their variance $[F, \sigma(F)]$ from crystal samples, the exact details of the diffraction experiment depending on the experimental objectives (for example, collection to a required resolution limit at a specific wavelength in order to take advantage of anomalous scattering). The intensity diffracted by a crystal rotating in an X-ray beam can be expressed (Drenth, 2001) as
(C) 2006 International Union of Crystallography Printed in Denmark - all rights reserved

$$
I=\left(\frac{\lambda}{w V^{2}}\right)^{3}\left(\frac{e^{2}}{m c^{2}}\right) V_{\mathrm{cr}} I_{0} L P T|F(h k l)|^{2} .
$$

In this equation, $I$ refers to the intensity of the diffracted beam, $e$ the electronic charge, $m$ the electronic mass, $V_{\text {cr }}$ the volume of the crystal and $V$ the volume of the unit cell; $L$ and $P$ are the Lorentz and polarization correction factors and $T$ refers to the proportion of X-rays that are not absorbed by the crystal.

Here we refer to the above equation and discuss the potential errors in each term. On a synchrotron beamline the wavelength $(\lambda)$ will be known with a certain precision and supplied with a certain energy bandpass. The angular velocity of the crystal rotating in the X-ray beam $(w)$ will be determined by the perfection of the electromechanical system used for rotating the crystal and the way the crystal is supported on this rotation axis (for example, a crystal supported in a 'cryoloop' mounted on a magnetic support). The beam intensity $I_{0}$ is given by the performance of the synchrotron and optics: any variation in this term comes from the X-ray optics and any relative movement of the beam with respect to the crystal (this include movements of the crystal in the cold nitrogen-gas stream, goniostat and beam stability). The $F$ values can change during an experiment owing to the crystal's decay in the beam with radiation dose. Most of the terms in the above equation may hence be regarded as complicated functions of time. Small errors in these parameters are typically taken into account by empirical models in data scaling.

The major elements (source, optics, goniostat) which influence the above equation are now discussed moving from the
Received 2 March 2005

Accepted 25 November 2005 
source to the sample (detailed discussions on detectors and radiation damage are the subject of other papers in this issue), their operation and state-of-the-art performance are explained and some likely future developments are presented. An approach to monitoring temporal variations in the quantities that condition the above equation is proposed, leading to the concept of quality control of data collected on a synchrotron-radiation source.

\section{Modern synchrotron-radiation sources}

In the late 1970s, parasitic usage of synchrotron radiation from rings constructed for high-energy physics (and nearing the end of their usefulness for that purpose) demonstrated clearly that a synchrotron is an extremely useful and flexible source of radiation for the study of matter. The pioneering synchrotronradiation facilities at (for example) DESY, LURE, NINA, SPEAR and ADONE were quickly replaced by 'storage rings' dedicated to providing synchrotron radiation from bending magnets over a broad spectral range and with long continuous periods of beam. Such rings were designed and constructed at Daresbury (SRS), Brookhaven (NSLS) and Tsukuba (Photon Factory). All these sources (which came on line in quick succession in the early 1980s) are still operational and highly productive. The availability of such sources provided the stimulation for an ever-increasing and international synchrotron user community, crying out for stronger and more brilliant beams ${ }^{1}$. Table 1 shows a list of several notable synchrotron sources that are operational or under construction: a more complete list has been compiled by Winick (2000).

At the beginning of the 1990s, the first 'low-emittance' storage rings were constructed using relatively high-energy (and hence large) rings but decreasing the emittances previously obtained by more than an order of magnitude. These large-circumference rings (ESRF, APS and SPring-8) permitted the construction of long straight sections into which insertion devices ${ }^{2}$ could be installed. The range of beam requirements of different types of experiment stimulated the development of a wide variety of insertion devices.

\subsection{Undulators as sources of synchrotron radiation}

Most recent macromolecular (MX) beamlines have been constructed using an undulator as radiation source, since undulators are capable of producing highly brilliant beams but with a relatively low power loading on the beamline optics. An undulator is a device composed of a series of magnetic dipoles whose poles are reversed after each dipole so that the electron beam follows a series of (generally horizontal) wiggles. The maximum angular excursion of electrons in the wiggle is $K / \gamma$,

\footnotetext{
${ }^{1}$ Brilliance is photon flux per $\mathrm{mm}^{2}$ per $\operatorname{mrad}^{2}$ per $0.1 \%$ energy bandwidth of the beam: a brilliant beam is both highly parallel and has a tiny 'source size'. Brilliance is directly linked to the property of a storage-ring electron beam called 'emittance', which describes the size and angular trajectory of the circulating electron beam.

${ }^{2}$ Insertion devices are magnetic elements inserted into straight sections which locally modify the electron-beam trajectory and hence can produce synchrotron radiation with different characteristics to that emitted by a bending magnet.
}

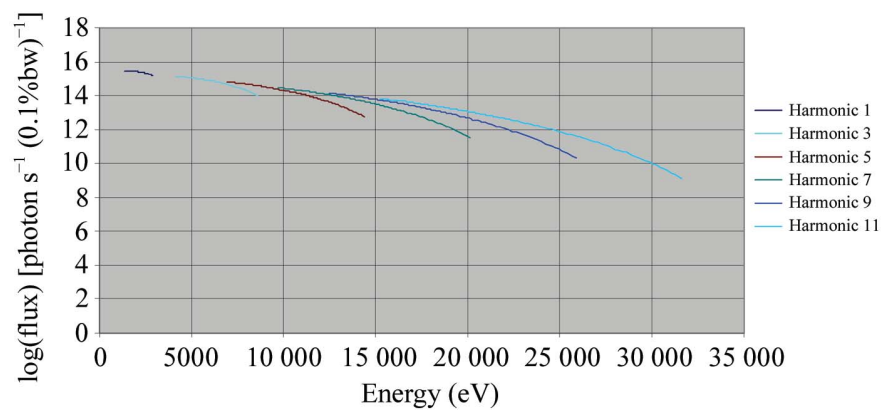

Figure 1

An undulator tuning curve calculated for the SOLEIL synchrotron $(2.75 \mathrm{GeV}, 500 \mathrm{~mA}$ stored current) and a $20 \mathrm{~mm}$ period in-vacuum undulator. The solid curves correspond to the range of energies traced out by each undulator harmonic as the gap is changed. Small gap values correspond to low energies. Only the strongest (odd) harmonic tuning curves are shown. The curve was calculated using the program SPECTRA (Kitamura et al., 2004).

where $K$ is known as the deflection parameter and $1 / \gamma$ is the opening angle of radiation produced by the electron beam (which depends on the storage-ring energy $E$ ). When $K \sim<1$, then the beam-deflection angle is less than the natural opening angle of the radiation beam, thus allowing the radiation from different 'nodes' to interfere (see, for example, Krinsky, 1983; Walker, 1986; Kim, 1995). The radiation then appears as a series of intense maxima (harmonics) which can be shifted in energy (tuned) by changing the gap between the two sets of magnetic poles (and hence changing $K$ ),

$$
\begin{gathered}
K=0.934 \lambda_{u} B_{0}, \\
\lambda_{1}=\frac{13.056 \lambda_{u}}{E^{2}}\left(1+\frac{K^{2}}{2}\right) .
\end{gathered}
$$

In the above equations $E$ is the storage-ring energy $(\mathrm{GeV}), \lambda_{1}$ is the wavelength $(\AA)$ of the undulator first harmonic, $\lambda_{u}$ is the undulator period in $\mathrm{cm}, K$ is the undulator deflection parameter and $B_{0}$ is the undulator field strength.

The relation between the $K$ and the wavelength of harmonic peaks in the undulator spectrum can be understood by examining these equations. For example, increasing the field strength by closing the undulator gap (making the upper and lower magnetic arrays closer) increases $K(2)$. An increased $K$ also increases the wavelength of the first undulator harmonic (3), so the undulator spectrum shifts to lower energy when the undulator gap is closed. If we are interested in the hard X-ray region typically used by crystallographers $(5-20 \mathrm{keV}, 2.5-$ $0.8 \AA$ ), then these energies are made accessible by decreasing the undulator period or increasing the storage-ring energy. We can note that hard X-rays (short wavelengths) are more easily accessible on the high-energy storage rings $\left(1 / E^{2}\right.$ dependance of equation 3). An undulator tuning curve for a $20 \mathrm{~mm}$ period in a vacuum undulator at SOLEIL is given in Fig. 1. This curve shows the energies and beam intensity traced out by the peak of the odd harmonics as the undulator gap is opened ( $K$ decreased).

The higher energy of storage rings such as ESRF, APS or SPring-8 ensure that with permanent-magnet insertion devices 
Table 1

A list of some of the principal synchrotron radiation sources where MX beamlines have been built, showing a huge improvement in electronbeam emittance over the last $25 \mathrm{y}$.

\begin{tabular}{lllllll}
\hline & & & & \multicolumn{2}{l}{ Emittance (nm rad) } \\
\cline { 6 - 7 } Machine & $\begin{array}{l}\text { Start of user } \\
\text { operation }\end{array}$ & $\begin{array}{l}\text { Energy } \\
(\mathrm{GeV})\end{array}$ & $\begin{array}{l}\text { Current } \\
(\mathrm{mA})\end{array}$ & Horizontal & Vertical \\
\hline DORIS & $1981(1991)$ & 4.4 & 120 & 400 & 10 \\
SRS & 1981 & 2.0 & 200 & 100 & $\sim 1$ \\
NSLS & 1983 & 2.8 & 280 & 45 & 0.1 \\
ESRF & 1995 & 6 & $200(250)$ & 4 & 0.03 \\
APS & 1997 & 7 & 300 & 3 & 0.1 \\
SPring-8 & 1997 & 8 & 100 & 3 & 0.07 \\
ELETTRA & 1995 & 2 & 300 & 7 & 0.007 \\
ALS & 1995 & 1.9 & 400 & 6.8 & 0.15 \\
MAX II & 1996 & 1.5 & 300 & 8.8 & 0.09 \\
BESSY II & 1998 & 1.9 & $200(400)$ & 6 & 0.04 \\
SLS & 2001 & 2.4 & 400 & 5 & 0.04 \\
SOLEIL & $\sim 2006$ & 2.75 & 500 & 3.7 & 0.037 \\
DIAMOND & $\sim 2006$ & 3 & 300 & 2.7 & 0.03 \\
\hline
\end{tabular}

hard X-rays (10-20 keV) are accessible even on relatively low harmonics (first, third, fifth) of undulator sources. The development of in-vacuum undulators (Yamamoto et al., 1992; Kitamura, 1998) has increased the range of tunability of shortperiod undulators, hence allowing further exploitation of the hard X-ray region and accessibility to wavelengths commonly used for MX even on the intense first harmonic.

Most recently, these storage-ring improvements (improved emittance, the use of short periods, in-vacuum undulators) have been incorporated into the design of several 'intermediate energy sources' (Corbett \& Rabadeau, 2003) such as SLS, SOLEIL, ALBA and Diamond (the latter three sources being under construction). These sources preserve most of the advantages of the large 'high-energy' synchrotron facilities, whereas they add 'cheapness and compactness' to the list of desirable properties.

\subsection{Future directions for sources of synchrotron radiation for $M X$}

Work continues on improvements to synchrotron-radiation sources. Recently, proposed storage rings or upgrades include (for example) the MAX IV project at MAX-LAB in Lund, NSLS II at Brookhaven and the PETRA III upgrade at DESY in Hamburg, both of which aim to be producing beams with emittances of $1 \mathrm{~nm}$ rad or less in the next $5 \mathrm{y}$, which will give a further leap in beam brilliance. The design of an ultimate $\mathrm{X}$-ray storage-ring light source has been discussed by Elleaume \& Ropert (2003). In terms of insertion devices, several groups are engaged on research projects to increase the field strength of undulator magnets and hence obtain hard $\mathrm{X}$-rays from medium-energy sources at low harmonic numbers. These projects include the development of superconducting undulators (Kubsky et al., 2003) and the lowtemperature undulators (Hara et al., 2004). For a review of the advantages and disadvantages of cryogenic versus lowtemperature undulator sources, see Kitamura et al. (2004).

\section{Optical components for undulator beamlines}

Beamline optical components (setting apart simple equipment such as beam-defining slits or apertures) belong to two main categories: crystal optics (for monochromatization of the X-ray beam but also used for focusing) and grazing-incidence mirror optics (for focusing the X-rays). The following discussion is meant to give an overview (discussions of multilayer mirrors, various methods of microfocusing beams, exotic types of monochromator etc. are not covered).

\subsection{Monochromators and cryogenic cooling}

Crystal monochromators select a single energy from a polychromatic X-ray beam according to Bragg's law. The energy bandpass of these monochromators depends on the natural width of the diffracted beam (the 'rocking width' or 'Darwin width') and the photon-beam divergence accepted by the monochromator crystal. Several crystal monochromator materials have been used at synchrotron MX beamlines, but by far the most common is silicon (because of its ready availability in a high-purity form). Crystals can be cut and polished to diffract from different sets of Bragg planes according to experimental bandpass requirements. There are a multitude of specific monochromator geometries for different functions, but those most commonly found on MX beamlines are described below.

(i) A single crystal, sometimes with the crystal surface cut at an angle to the principal Bragg planes (asymmetric cut crystal). This has the obvious disadvantage that the output beam is diffracted at a considerably different angle for differing energies [for example, $9.8^{\circ}$ for the Se absorption edge and $17^{\circ}$ for the Fe absorption edge for an Si (111) monochromator] and hence the experiment has to move to accommodate wavelength change. Examples of the use of such monochromators are given in Lemonnier et al. (1978) and Helliwell et al. (1982).

(ii) The 'simple' channel-cut monochromator crystal consisting of two reflecting surfaces cut from a monolithic Si block, the second surface being used to retransmit the beam parallel to the incident beam. This type of crystal, described, for example, in Materlik \& Kostroun (1980), Berman et al. (2002) and Zhang et al. (2003), has many great advantages (not least its simplicity) but one main disadvantage, this being that the separation between the output and input beams depends on the Bragg angle of the monochromator (the offset is $2 D \cos \theta$, where $D$ is here the separation between the two reflecting surfaces). Various schemes have been developed to avoid this problem (see, for example, Oestreich et al., 1998).

(iii) A double-crystal monochromator preserves the same geometry (two reflecting surfaces), but the second surface is translated in order to keep a constant offset between the output and input beams. The inconvenience of this arrangement is the stringent requirement for mechanical and thermal stability of both crystal surfaces in order to maintain this constant offset. Good mechanical solutions to these problems exist, making this type of monochromator probably the most common in current usage. 
(iv) A sagitally focusing double-crystal monochromator, where the second crystal is curved in order to focus the beam in the horizontal direction (Freund, Comin et al., 1998; Schulze-Briese et al., 1998).

Monochromator crystals are always (on modern synchrotron sources) cooled against the power of the incoming X-ray beam. Since these devices are installed in a vacuum chamber, even small input-beam powers can cause significant heating (and hence deformation) of the surface of the crystal, causing energy offset, increased rocking curve and beam divergence. The decay of the synchrotron beam with time, the introduction of attenuators, the modification of beam slit sizes in front of the monochromator and the changing of an undulator gap all modify the power loading on the monochromator crystal and hence change its thermal equilibrium. Monochromator cooling systems thus need to cool efficiently and react rapidly to changing heat loads.

It was noted by Bilderback (1986) that at temperatures attainable using liquid-nitrogen cooling the coefficient of linear expansion of $\mathrm{Si}$ drops to zero. In fact, the size of the deformation of the surface of a monochromator crystal is proportional to $P \alpha / k$, where $\alpha$ is the coefficient of linear expansion, $k$ is the coefficient of thermal conduction (Freund, Gillet et al., 1998) and $P$ is the incident power of the beam. A figure of merit for the performance of a monochromator crystal at high power load is then $k / \alpha$. This figure increases sharply for $\mathrm{Si}$ at $80 \mathrm{~K}$ and this principle forms the basis of operation for many crystal monochromators in high-power synchrotron beams (Bilderback et al., 2000). The maximum power load supportable by such cryogenically cooled monochromators is discussed by Lee et al. $(2000,2001)$. Several lower $Z$ materials have been tested as monochromator crystals. In particular, diamond has a favourable figure of merit even at room temperature and the additional property of absorbing weakly in the hard X-ray region. Diamond is then a very attractive monochromator material, its main drawbacks being the availability of high-quality crystals and the small size of crystals available. Nonetheless, a number of successful monochromators using diamond have been installed at several synchrotron sources (see, for example, Fernandez et al., 1997; Burmeister et al., 1998; Freund, Sellschop et al., 1998). Development of high-quality synthetic diamond is a very active area of research for a number of applications which may improve this situation

Of particular interest for the future is the 'topping-up mode' operation developed at the Swiss Light Source and in operation at the APS (also planned for the DIAMOND and SOLEIL sources), where electron-beam decay is offset by injecting beam into the storage ring every few minutes and consequently maintaining a quasi-continuous power loading on beamline optics (and promoting thermal equilibrium).

\subsection{Mirror optics}

X-ray mirrors exhibit total external reflection at small incidence angles (grazing angles) of less than a degree. For a given graze angle, the mirror gives a 'high-energy cutoff', i.e. higher energy beams are not reflected. This cutoff can be modified by coating mirrors with high- $Z$ material. Mirror reflectivity is also modified by surface quality (for example, surface roughness and slope error).

Various optical configurations are used. Here, we concentrate on the Kirkpatrick-Baez (KB) optical arrangement, which is becoming increasingly used at synchrotron sources owing to the increasing brilliance of sources, the desire for controllable microfocusing and the improvements in recent years in the quality of mirrors produced by commercial suppliers.

The KB geometry is shown schematically in Fig. 2(b). Two separate mirrors focus in the vertical and horizontal: the two foci are then independently adjustable allowing (in principle) the beam shape to be matched to the sample size. Sophisticated bending mechanisms (Eng et al., 1998; Dabin et al., 2002) and improved mirror-fabrication techniques have made it possible to bend mirrors to an elliptical form and hence achieve a close-to-perfect focus for arbitrary demagnification ratio. This has made it possible to strongly demagnify beams from the synchrotron source. Focal spots of several tens of micrometres (which ten years ago were the domain of specialist 'microfocus' beamlines) are now readily produceable with this type of optics and sub-micrometre focusing of $\mathrm{X}$-rays is being exploited at a number of sites. Diffractionlimited focal spots beckon! The protein crystallographer then has a wider range of choice of optical tool. Large low-divergence beams can be generated either to study crystals with huge unit-cell parameters or to limit the flux density of the beam on the crystal (and hence slow down the onset of radiation damage). Alternatively, microfocus beams with large divergences but which are able to illuminate small fractions of a crystal volume (either for diffraction-quality reasons or in order to illuminate a 'fresh' part of a larger crystal) are readily produced. Such a microfocus protein crystallography facility (based on mirror and crystal monochromator optics) is already available at the SLS, small focus spots are available at the APS, ESRF (ID29 and ID23) and microfocus facilities using KB optics are under development at SOLEIL, DIAMOND, APS and the ESRF.

Wavefront-analysis techniques help to establish almost ideal focal spots. In this method, the form of curvature of the mirror is matched to that required for a near-perfect focus. The effect of mirror benders on the overall shape of the mirror is studied and the required bending conditions to correct deformations to the focus (caused, for example, by modification of some rays' trajectories by the presence of a thermal bump on a crystal monochromator) can be corrected and rays from the source restored to their optimal path. This technique has been applied to KB optics by Hignette et al. (1997) in the $\mathrm{X}$-ray region and Mercre et al. (2003) in the extreme ultraviolet.

A refinement of this scheme is proposed by Signorato et al. (2001), using so-called bimorph mirrors which are constructed of a reflecting surface underlaid by multiple piezo-actuators that allow local modification of the form of the mirror surface. This gives a significant increase in the frequency range of 


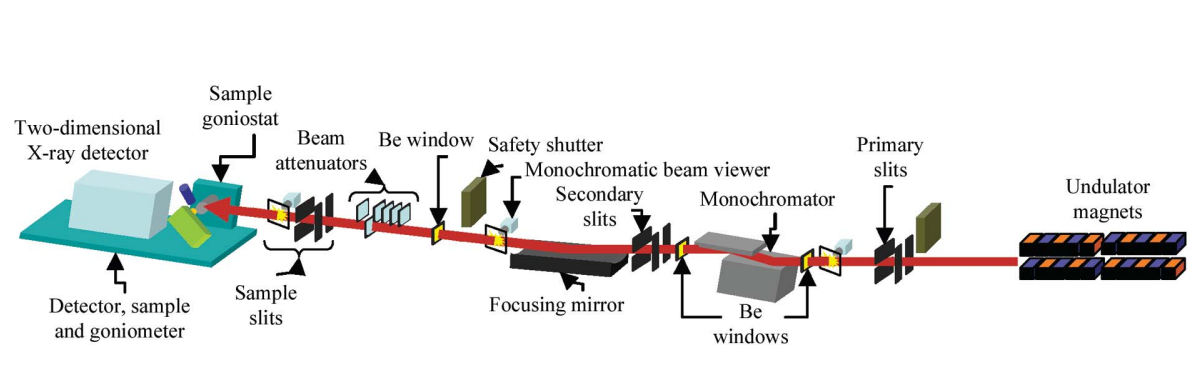

(a)

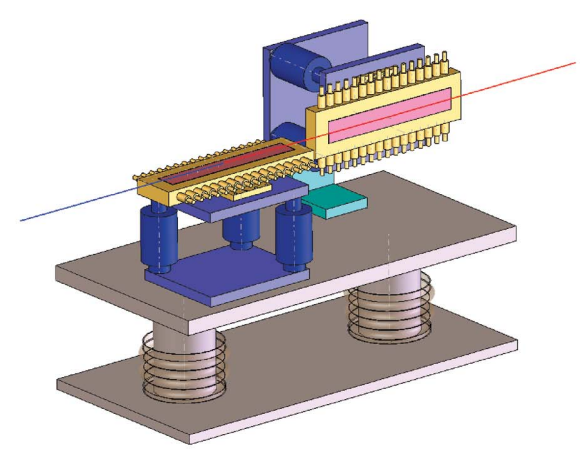

$(b)$

Figure 2

(a) A highly schematic diagram showing a synchrotron beamline from undulator to sample. Many variants of the optical theme exist [focusing by separated vertical and horizontal mirrors as in $(b)$, pre-focusing and post-focusing mirrors, focusing monochromators etc.]. (b) A schematic drawing of the installation of a Kirpatrick-Baez mirror pair on the SOLEIL PROXIMA 1 beamline. The beam enters from the right-hand side of the drawing and is successively focused in the horizontal (vertically orientated mirror) and vertical directions.

defects in the wavefront that can be corrected. Such bimorph mirrors have been installed on the GM/CA beamline at the APS and are being installed on beamlines I02, I03 and I04 at DIAMOND and the PROXIMA 1 beamline at SOLEIL.

\section{Goniostats, sample-changing robots and automation of measurements}

Goniometry that allows the easy handling and alignment of microcrystals has been the object of much recent development work. The use of sample-viewing optics through which the $\mathrm{X}$-ray beam is allowed to pass (and associated alignment facilities) pioneered by Cipriani and coworkers (Perrakis et al., 1999; Riekel, 2004) and incorporated in the ESRF/EMBL microdiffractometer has made it easier to manipulate tiny crystals (less than $10 \mu \mathrm{m}$ in the longest dimension). Increased usage of air-bearing rotation axes (for example, at the ALS, SLS and ESRF) has ensured that cylinders of confusion of a few micrometres can be achieved with rotations around a single axis. Sample-orienting systems such as the mini kappa device under development at the ESRF/EMBL or the full miniaturized kappa goniostat developed by Rock and Rosenbaum (described in Fischetti et al., 2004) allow increased choice of sample alignment prior to data collection around a precise rotation axis. Software has been developed to allow such goniometers to work in conjunction with several samplechanging robot mechanisms which have been developed in recent years [see, for example, Cohen et al. (2002); Ohana et al. (2004); Ueno et al. (2004) and the commercially available ACTOR, BruNo and MAR Research devices]. These all operate in conjunction with the goniostat and a liquidnitrogen reservoir to allow a sample to be transferred from a puck (brought to the beamline in a transport Dewar) to the goniostat, generally speaking using magnetic bases and pins to attach the crystal, allowing the possibility of a data-collection 'pipeline' with automatic submission and performance of an experiment to be monitored at a distance (http://clyde.dl.ac.uk/ e-htpx/index.htm, http://www.dna.ac.uk/index.html).

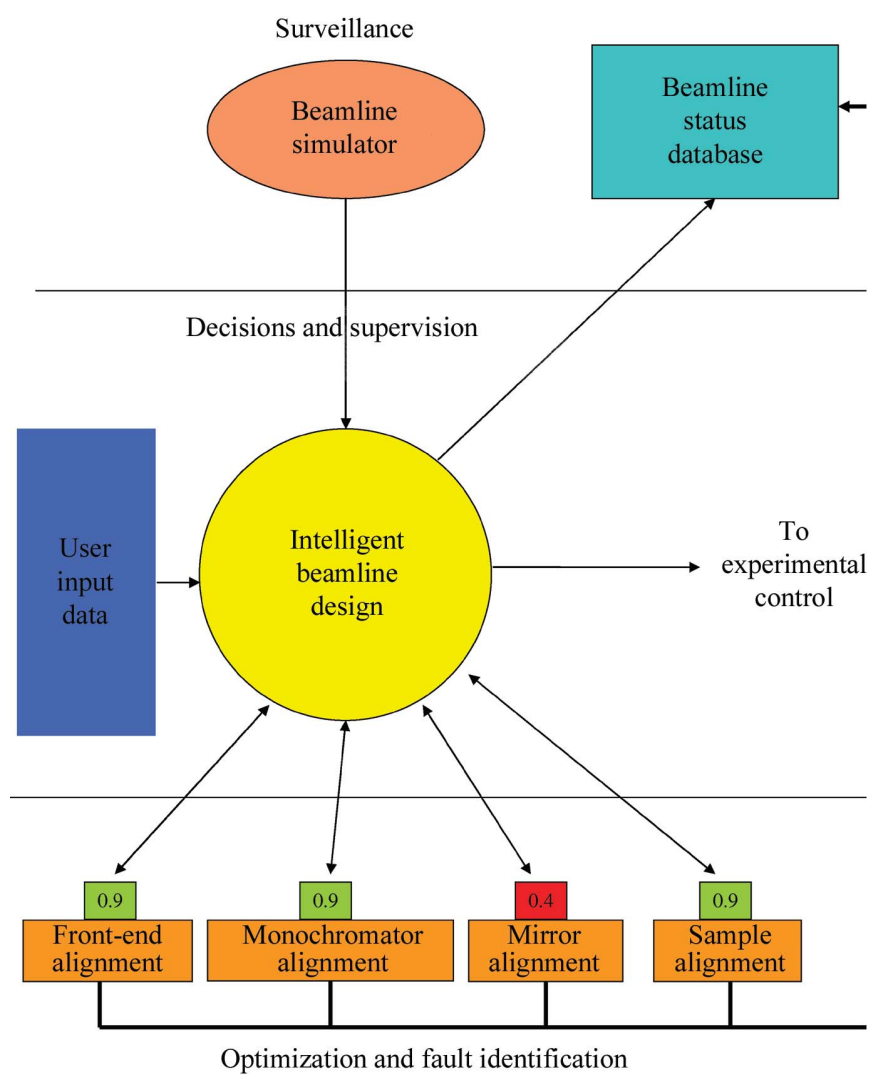

Figure 3

A schematic block diagram to show how a beamline fault might be localized (and hence diagnosed and corrected). The surveillance system receives information on the status of the beamline (lower part of the drawing). The intelligent beamline device compares the expected beamline performance for each sub-assembly (either by prior knowledge of expected performance or by modelling) in order to verify the state (denoted here schematically by a 'figure-of-merit value') of each subassembly. This process can be extended to identify individual faulty components.

From the above discussion, it can be seen that extremely brilliant beams can be brought to bear on crystal samples with increasing control and automation in the alignment of optical systems, so that close to ideal conditions can be maintained at 
the sample position. In addition, automated end stations and highly evolved software can take routine decisions and are opening the way up to 'beamline measurements without users'. This tendency implies an increased need for automatic supervision of the 'state of health' of the synchrotron beamline, which is turn is necessary to ensure experimental quality control.

A particular challenge is that beamline supervision must operate over a large range of timescales. Indeed, the timescale of a reflection passing through the Ewald sphere is in the millisecond to tens of milliseconds range, the time for the collection of a diffraction image is in the range of seconds to several seconds, the time for an experiment is of the order of minutes and the time for a sequence of experiments (phasing plus high resolution plus mutants) is several hours. However, MX experiments tend to use integrating detectors as the easiest means to efficiently collect large numbers of diffraction spots and hence the faster timescale events are also integrated.

The problem of automated beamline supervision is currently being studied at SOLEIL. One approach, investigated in conjunction with the DREAM (Diagnosing, Recommending Actions and Modelling) team from IRISA in Rennes, is to link a specific fault state (for example, a beam instability) to the observable effects this state will (or could) cause on the beamline. Such links, represented by causal graphs (see, for example, Giarratano \& Riley, 1998), can then be analysed using abductive logic rules allowing the development of a set of hypotheses about the fault which caused a given symptom to be observed.

However, when building the model of a complex system, it often turns out to be important to take into account its architecture and/or functional topology. For a synchrotron beamline such topology can be said to be linear, notably in the sense that the change in beam characteristics (which provide the main criteria for distinguishing between correct and faulty behaviour) propagates in the direction from the source to the sample. This allows fault-source isolation at a coarse level, i.e. between subsystems such as monochromator, mirror etc., as shown in Fig. 3. Causal graph formalism may then not be the most appropriate way to describe the functional dependence of a whole beamline, but could be efficient in analysing each coarse subsystem.

Whatever the formalism (model) chosen to represent the beamline and its equipment, in order to link a series of 'beamline symptoms' to an identifiable fault condition, sufficient indicators of the fault condition need to be identified and corresponding detection systems installed in the beamline. A model-based approach then leads both to a method of identifying faults and to a description of necessary beamlinemonitoring equipment (and where this equipment should be installed on a beamline).

The problem then becomes one of being able to install sufficient beam-monitoring equipment in a beamline and to be able to make assessments over an extended time range as described in the previous paragraph. In this way, a suitable software could intervene in a data collection when the beamline has moved away from its optimal performance for a given experiment. Such an intervention could be simply warning the user of non-optimal beam conditions, the flagging of data collected during poor beam conditions or eventually the use of sophisticated beamline-alignment algorithms (for example, those used for wavefront analysis described above) to take corrective action. The latter, however, remains a very long-term goal!

An alternative approach to the problem of supervising and correcting the state of a beamline is to compare actual beamline status (as measured by various beam position and profile monitors) to theoretical beamline status [as calculated with a ray-tracing program such those developed by Moreno (2002) or Svensson (2004)]. Note that such a calculation needs to be remade with every modification to the beamline (for example, change of slit size, mirror position or angle, wavelength, undulator gap) and needs to be performed with measured mirror parameters (form of the mirror surface measured at various points, for example). The calculation must then be made quickly and with sufficient efficiency of rays as to give a good statistical model of beam form at the required point in the beamline (in this context 'efficiency' refers to the loss of traced rays along the modelled beamline). Obviously tracing rays costs computer calculation time and many rays are 'lost' to the calculation by being stopped by slits, not being reflected or diffracted by an optical component etc. Such an approach is being attempted at SOLEIL for a simple 'sub-problem' of an undulator source and set of beamline slits. The advantage of such an approach can be seen schematically in Fig. 3. A comparison made between model and reality allows problems to be traced to particular subsystems or parts of the beamline.

\section{Conclusions}

Modern synchrotron instrumentation from the undulator X-ray beam sources through the optics and down to the goniostat have been described and several possible future improvements discussed. The possibility of using tools from the world of artificial intelligence (model-based analysis and causal graph analysis) in order to verify correct beamline performance at any particular time is discussed.

OR is supported under the European Commission Sixth Framework Programme Integrated Project BIOXHIT. The authors wish to thank Thierry Moreno and Oleg Chubar of SOLEIL for helpful discussions, the ESRF Macromolecular Crystallography Group for measurements and discussions enabling the ongoing development of automated beamline supervision and the EMBL Grenoble Outstation instrumentation group for inspiration and helpful collaboration.

\section{References}

Berman, L. E., Siddons, D. P., Montanez, P. A. \& Lenhard, A. (2002). Rev. Sci. Instrum. 73, 1481-1484.

Bilderback, D. H. (1986). Nucl. Instrum. Methods Phys. Res. A, 246, 434-436. 
Bilderback, D. H., Freund, A. K., Knapp, G. S. \& Mills, D. M. (2000). J. Synchrotron Rad. 7, 53-60.

Burmeister, W. P., Bourgeois, D., Kahn, R., Belrhali, H., Mitchell, E. P., McSweeney, S. M. \& Wakatsuki, S. (1998). Proc. SPIE, 3448, 188196.

Cohen, A. E., Ellis, P. J., Miller, M. D., Deacon, A. M. \& Phizackerley, R. P. (2002). J. Appl. Cryst. 35, 720-726.

Corbett, J. \& Rabadeau, T. (2003). Nucl. Instrum. Methods Phys. Res. $A, \mathbf{5 0 0}, 11-17$.

Dabin, Y., Rostaing, G., Hignette, O., Rommeveaux, A. \& Freund, A. K. (2002). Proc. SPIE, 4783, 235-245.

Drenth, J. (2001). International Tables for Crystallography, Vol. F, edited by M. G. Rossman \& E. Arnold, pp. 45-63. Dordrecht: Kluwer Academic Publishers.

Elleaume, P. \& Ropert, A. (2003). Nucl. Instrum. Methods Phys. Res. $A, \mathbf{5 0 0}, 18-24$.

Eng, P. J., Newville, M., Rivers, M. L. \& Sutton, S. R. (1998). Proc. SPIE, 3449, 145-156.

Fernandez, P. B., Graber, T., Lee, W.-K., Mills, D. M., Rogers, C. S. \& Assoufid, L. (1997). Nuclear Instrum. Methods Phys. Res. A, 400, 476-483.

Fischetti, R. S., Stepanov, S., Rosenbaum, G., Barrea, R., Black, E., Gore, D., Heurich, R., Kondrashkina, E., Kropf, A. J., Wang, S., Zhang, K., Irving, T. C. \& Bunker, G. B. (2004). J. Synchrotron Rad. 11, 399-405.

Freund, A. K., Comin, F., Hazemann, J.-L., Hustache, R., Jenninger, B., Lieb, K. \& Pierre, M. (1998). Proc. SPIE, 3448, 144-155.

Freund, A. K., Gillet, J.-A. \& Zhang, L. (1998). Proc. SPIE, 3448 , 362-372.

Freund, A. K., Sellschop, J. P., Lieb, K., Rony, S., Schulze-Briese, C., Schroeder, L.-E. \& Teyssier, J. (1998). Proc. SPIE, 3448, 5363.

Giarratano, J. \&. Riley, G. (1998). Expert Systems: Principles and Programming. PWS Publishing Company.

Hara, T., Tanaka, T., Kitamura, H., Bizen, T., Marechal, X., Seike, T., Kohda, T. \& Matsuura, Y. (2004). Phys. Rev. Special Topic Accelerator Beams, 7, 050702.

Helliwell, J. R., Greenhough, T. J., Carr, P. D., Rule, S. A., Moore, P. R., Thompson, A. W. \& Worgan, J. S. (1982). J. Phys. E, 15, 13631372.

Hignette, O., Freund, A. K. \& Chinchio, E. (1997). Proc. SPIE, 3152, 188-199.

Kim, K.-J. (1995). Opt. Eng. 34, 342-352.

Kitamura, H. (1998). J. Synchrotron Rad. 5, 184-188.
Kitamura, H., Hara, T., Tanaka, T., Bizen, T., Marechal, X. \& Seike, T. (2004). Proceedings of EPAC 2004, Lucerne, Switzerland, p. 59. European Physical Society Accelerator Group.

Krinsky, S. (1983). Brookhaven National Laboratory Report, pp. 1-5. Kubsky, S., Dolling, D., Geisler, A., Hobl, A., Klein, U., Krischel, D., Moser, H., Rossmanith, R. \& Couhan, S. (2003). In Proceedings of the Workshop on Superconducting Undulators and Wigglers, ESRF, Grenoble, France.

Lee, W.-K., Fernandez, P. \& Mills, D. M. (2000). J. Synchrotron Rad. 7, $12-17$.

Lee, W.-K., Fezzaa, K., Tajiri, G. \& Mills, D. (2001). J. Synchrotron Rad. 8, 22-25.

Lemonnier, M., Fourme, R., Rousseau, F. \& Kahn, R. (1978). Nuclear Instrum. Methods Phys. Res. 152, 173-177.

Materlik, G. \& Kostroun, V. O. (1980). Rev. Sci. Instrum. 51, 86-94.

Mercre, P., Zeitoun, P., Idir, M., Le Pape, S., Douillet, D., Levecq, X., Dovillaire, G., Bucourt, S., Goldberg, K. A., Naulleau, P. P. \& Rekawa, S. (2003). Opt. Lett. 28, 1534-1536.

Moreno, T. (2002). SpotX for Microsoft Windows 95. Paris: Caminotec Scientific Software.

Oestreich, S., Kaulich, B., Barret, R. \& Susini, J. (1998). Proc. SPIE, 3448, 176-187.

Ohana, J., Jacquemet, L., Joly, J., Bertoni, A., Taunier, P., Michel, L., Charrault, P., Pirocchi, M., Carpentier, P., Borel, F., Kahn, R. \& Ferrer, J.-L. (2004). J. Appl. Cryst. 37, 72-77.

Perrakis, A., Cipriani, F., Castagna, J.-C., Clasutre, L., Burghammer, M., Riekel, C. \& Cusack, S. (1999). Acta Cryst. D55, 1765-1770.

Riekel, C. (2004). J. Synchrotron Rad. 11, 4-6.

Schulze-Briese, C., Heidenreich, G., Auderset, H., Vermeulen, D. \& Freund, A. K. (1998). Proc. SPIE, 3448, 156-165.

Signorato, R., Carre, J.-F. \& Ishikawa, T. (2001). Proc. SPIE, 4501, 76-87.

Svensson, O. (2004). Personal communication.

Ueno, G., Hirose, R., Ida, K., Kumasaka, T. \& Yamamoto, M. (2004). J. Appl. Cryst. 37, 867-873.

Walker, R. P. (1986). Synchrotron Radiation for X-ray Crystallography, edited by M. Hart, pp. C1-C28. Erice, Italy: Ettore Majorana Centre for Scientific Culture.

Winick, H. (2000). Synchrotron Radiat. News, 13, 38-39.

Yamamoto, S., Shioya, T., Hara, M., Kitamura, H., Zhang, X. W., Mochizuki, T., Sugiyama, H. \& Ando, M. (1992). Rev. Sci. Instrum. 63, 400-403.

Zhang, L., Lee, W. K., Wulff, M. \& Eybert, L. (2003). J. Synchrotron Rad. 10, 313-319. 